\title{
Design and synthesis of new hybrid latency reversal agents for HIV treatment
}

\section{Juliana Romano Lopes ${ }^{1}$, Igor Prokopczyk ${ }^{1}$, Jean Leandro dos Santos ${ }^{1}$}

${ }^{1}$ State University of São Paulo - UNESP, School of Pharmaceutical Science, Araraquara, São Paulo, Brazil, 14800-903

\section{Background}

The HIV-1 latent reservoir remains one of the main barriers to eliminate the virus. The latent reservoir is established after activated CD4+ T cells containing integrated proviral DNA differentiates into a resting memory cells with reduced transcriptonal activity. ${ }^{1}$ A strategy named "kick-and-kill" (also known as "shockand-kill") has emerged as an alternative to purge HIV-1 from reservoir in the first step, and after, to kill it using vaccines/antibodies or even immune system activation. ${ }^{2}$ Latency reversal agents (LRA) are an extensive class of compounds that can disrupt HIV latency. Among the classes of LRA, is possible to highlight the histone deacetylase (HDAC) and bromodomain (BRD) inhibitors. ${ }^{1,2}$ The potential of HDAC and BRD inhibitors to reactivate HIV-1 from latent reservoir was demonstrated in vitro and in vivo. ${ }^{3} 2$-aminobenzamide derivatives, as HDAC class I, and BRD4 inhibitors as those containing oxazole subunit has shown has been described to reactivate latent HIV-1 reservoir. ${ }^{4}$ The combination of LRA can promote synergism and induce a more promising reactivation in the latent reservoir. ${ }^{5}$ Based on these finds, we used molecular modification (hybridization) approach to describe the design, synthesis and evaluation of optimized hybrid compounds designed to target both BRD4 and HDAC class I (HDAC1-3).

\section{Methods}

All designed compounds were docked against BRD4 and HDAC2 in order to identify those more prone to interact with both targets at appropriate level (Figure 1). The more promising compounds were synthesized and evaluated through enzymatic assays using both HDAC and BRD enzymes. Molecular docking. Docking simulation was performed using Glide on Extra-precision mode by Maestro (Schrödinger ${ }^{\circledR}$ ) using BRD4 (PDB code: 4WIV; resolution $1.56 \AA$ ) and HDAC-2 (PDB code: 4LY1; resolution $1.56 \AA$ ) $)$. The pdb files were imported into the Maestro and prepared using the Protein Preparation Wizard. The interaction box ("grid") was defined by the Receptor Grid Generation, with dimensions of 10 $\AA$ × $10 \AA$ x $10 \AA$. All ligands were prepared using Ligand Preparation (LigPrep) with the OPLS3 force field and ionization states at $\mathrm{pH} 7 \pm 2$. Redocking studies were performed in order to validate the model. Synthesis. All compounds were prepared through divergent route, using classic organic reactions, according Scheme 1 and 2. Enzymatic assays. The ability of all compounds (at $10 \mu \mathrm{M}$ ) to inhibit both enzymes HDAC 1-3 and BRD4 were evaluated.
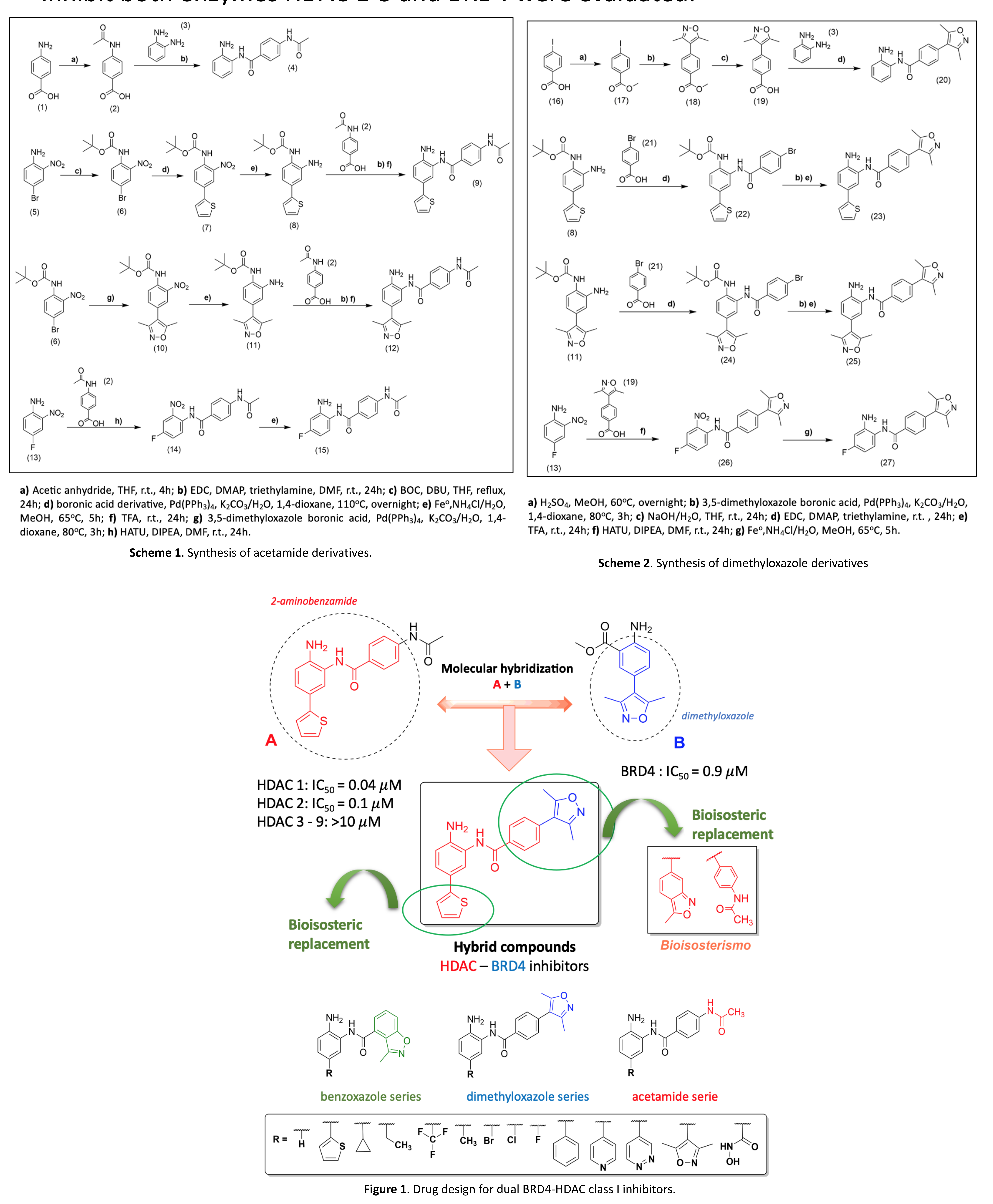

\section{Results}

The 8 more promissory compounds able to interact with both BRD-4 and HDAC2 through docking simulation were selected. The acetamide and 3,5dimethyloxazole derivatives were synthesized at yields ranging from $46-72 \%$. All compounds were characterized by analytical methods such as NMR, infrared, mass spectrometry and elemental analysis. The enzymatic assays (Table 1) reveals that compounds (4), (20), (9) and (23) inhibited HDAC class I, specifically HDAC 1-3 with values ranging from 75-89\% for HDAC1, 72-91\% for HDAC2 and 16-77 for HDAC3. Against BRD4, the inhibitory effect ranged from 5-25\%. The presence of 3,5-dimethyloxazole improved the recognition by bromodomain-4. All hybrids exhibited superior activity as HDAC inhibitor than BRD4 inhibitor.

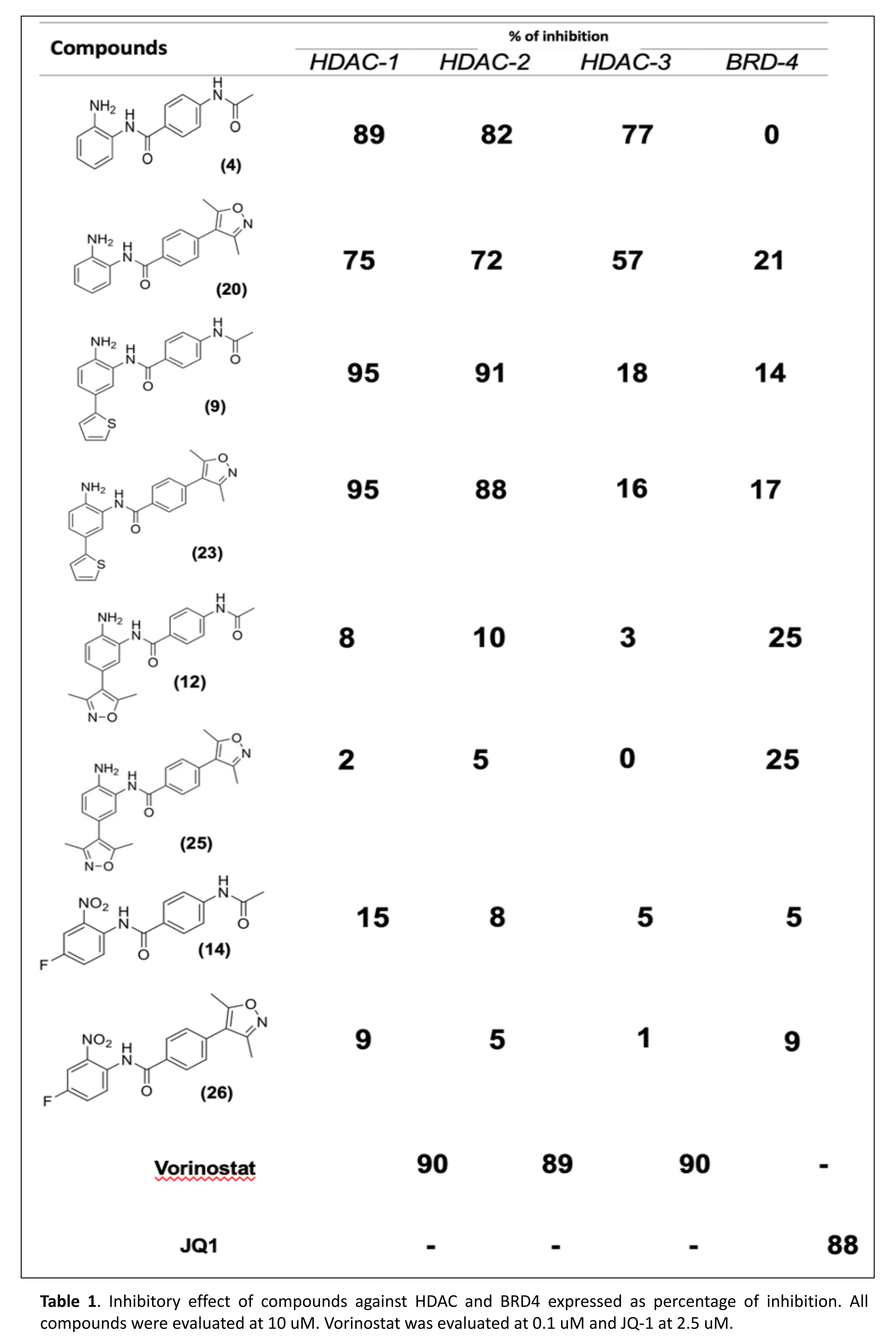

Conclusions

New hybrid HDAC/BRD4 inhibitors were synthesized and characterized by analytical methods. Docking studies contributed to selected the promising molecules for the synthesis. All of those were evaluated against the enzymes HDAC and BRD4. These compounds were able to inhibit BRD4 and HDAC 1-3 (class I) suggesting its potential use as latency-reversing agents. In the next steps, these compounds will be evaluated in vitro against infected HIV cells in order to characterize its latency-reversing effects.

\section{References}

1. Bruner, K. M., Wang, Z., Simonetti, F. R., et al. A quantitative approach for measuring the reservoir of latent HIV-1 proviruses. Nature (2019), 566: 120-125.

2. Lewin, S. R., Rasmussen, T. A. Kick and kill for HIV latency. Lancet (2020), 395(10227): 844-846

3. Lehrman, G., Hogue, I.B., Palmer, S., et al., Depletion of latent HIV-1 infection in vivo: a proof-of-concept study. Lancet (2005), 366: 549-555.

4. Banerjee, C. et al. BET Bromodomain Inhibition as a Novel Strategy for Reactivation of HIV-1. Journal of Leukocyte Biology (2012), 92(6): 1147-1154.

5. Abner E, Jordan A. HIV "shock and kill" therapy: In need of revision. Antiviral Research (2019), 166:19-34.

\section{Acknowledgments}

This study was supported by Fundação de Amparo à Pesquisa do Estado de São Paulo (FAPESP Ref. Process: FAPESP 18/11079-0); Programa de Apoio ao Desenvolvimento Científico da Faculdade de Ciências Farmacêuticas da UNESP - PADC. This study was financed in part by the Coordenação de Aperfeiçoamento de Pessoal de Nível Superior Brasil (CAPES) - Finance Code 001 and Conselho Nacional de Desenvolvimento Científico e Tecnológico (CNPq). J.L.S. is CNPq productivity fellows' level 2 (CNPq Ref. Process: $304731 / 2017-0)$. The funders had no role in study design, data collection and analysis. The authors declare not conflict of interests. 